\section{HUMANASE SOCIAIS \\ V.8 $\cdot$ N.2 • Agosto/Setembro/Outubro - 2019 \\ ISSN Digital: 2316-3801 \\ ISSN Impresso: 2316-3348 \\ DOI: $10.17564 / 2316-3801.2019 v 8 n 2 p 11-26$}
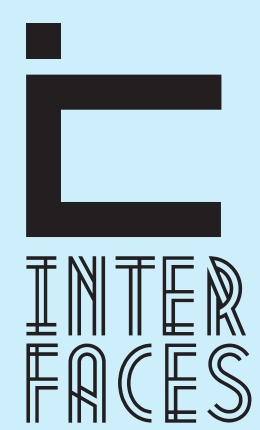

CIENTÍFICAS

\title{
REFLEXÕES SOBRE EXTENSÃO UNIVERSITÁRIA: UM ESTUDO DE CASO DO PROJETO “TESTE DA ORELHINHA EM IRATI E REGIÃO"
}

REFLECTIONS ON UNIVERSITY: A CASE STUDY OF THE PROJECT "NEONATAL HEARING SCREENNNG IN IRATI AND REGION"

REFLEXIONES SOBRE EXTENSIÓN UNIVERSITARIA: UN ESTUDIO DE CASO DEL PROYECTO "TEST DE LA OREJTA EN IRATI Y REGIÓN"

Karla Nadal ${ }^{1}$

Paulo Rogério Melo de Oliveira ${ }^{2}$

Cristiana Magni ${ }^{3}$

Marcos Roberto Kuhl ${ }^{4}$

\section{RESUMO}

Esta pesquisa identifica os benefícios gerados pelo projeto de Extensão Teste da Orelhinha em Irati e Região (TOIR) à comunidade local e traz reflexões sobre Extensão Universitária, a partir de suas políticas, ações e diretrizes institucionais. Trata-se de uma pesquisa aplicada, com abordagem qualitativa na qual se utilizou como instrumento para levantamento dos dados a aplicação de entrevista aberta semiestruturada, realizada com a comunidade atendida no projeto TOIR, professores, acadêmicos e gestores municipais de saúde. Foram empregados procedimentos metodológicos pautados em estudos descritivos e exploratórios como: levantamento bibliográfico, análise dos relatórios finais do projeto e pesquisa survey. Os resultados obtidos revelam que o principal benefício do projeto TOIR é a identificação da perda auditiva, antes de a criança completar três meses de idade e o encaminhamento dos bebês que falham na triagem ao médico otorrinolaringologista. Verifica-se também que o projeto estabelece uma relação entre a Universidade e outros setores da Sociedade, com vistas a uma atuação transformadora e implementadora de desenvolvimento regional e de políticas públicas. Além disso, a pesquisa revela que o projeto apresenta limites quanto ao acesso sobre os resultados dos encaminhamentos realizados para avaliação otorrinolaringológica. Por fim, aponta alguns caminhos que viabilizem a melhoria na execução do projeto.

\section{PALAVRAS-CHAVE}

Políticas Públicas. Extensão Universitária. Triagem Auditiva. 


\section{ABSTRACT}

This research identifies benefits generated by Permanent Extension project "Hearing Neonatal Screening" (HNS) in Irati and surrounding region, to the local community and brings reflections about University Extension from its policies, actions and Institutional guidelines. This is an applied research with a qualitative approach where a semi structured open questionnaire was used as an instrument to raise data carried out among the community assisted by the project such as teachers, academics and local administrators of public health. Methodological procedures were taken based on descriptive and exploratory procedures such as bibliographic survey and analysis of the final report of the project and survey. The results show that the main benefit of the project is the identification of the hearing loss before the child reaches the age of three months and the referral of the babies who fail the HNS to the otorhinolaryngologist (ENT doctor). The results were arranged in categories and the analysis reveals that the project's main benefit is the hearing loss identification to a diagnostic until the child is four months age. It seems that the project sets a relationship between the university and the society aiming to transform actions which meet interests and necessities of the population, implementing the local development of public policies. In addition, the research reveals that the project has limits to access the ENT doctor's diagnostics. This research shows some ways to improve the project's strategies.

\section{KEYWORDS}

Policies. University Extension. Hearing screening.

\section{RESÚMEN}

Esta investigación identifica los beneficios generados por el proyecto de Extensión Test de la Orejita en Irati y Región (TOIR) a la comunidad local y trae reflexiones sobre Extensión Universitaria, a partir de sus políticas, acciones y directrices institucionales. Se trata de una investigación aplicada, con abordaje cualitativo por el cual se utilizó como instrumento para el levantamiento de los datos la aplicación de encuesta abierta semiestructurada, realizada con la comunidad atendida en el proyecto TOIR, profesores, académicos y gestores municipales de salud. Se utilizaron procedimientos metodológicos pautados en estudios descriptivos y exploratorios como: levantamiento bibliográfico, análisis de los informes finales del proyecto e investigación survey. Los resultados obtenidos revelan que el principal beneficio del proyecto TOIR es la identificación de la pérdida auditiva, antes de que el niño cumpla tres meses de edad, y el encaminamiento de los bebés que fallan en la clasificación al médico otorrinolaringólogo. Se verifica también que el proyecto establece una relación entre la Universidad y otros sectores de la Sociedad, con vistas a una actuación transformadora e implementadora de desarrollo regional y de políticas 
públicas. Además, la investigación revela que el proyecto presenta límites en cuanto al acceso sobre los resultados de los encaminamientos realizados para evaluación otorrinolaringológica. Por último, apunta algunos caminos que viabilicen la mejora en la ejecución del proyecto.

\section{PALABRAS CLAVE}

Políticas Públicas; Extensión Universitaria; Prueba de audición.

\section{INTRODUCÇÃO}

A Universidade, enquanto Instituição social, influencia e sofre influência do contexto socioeconômico, político e cultural em que está inserida. Nesse sentido, o Ensino Superior tem o compromisso de estabelecer relações entre a Universidade e outros setores da Sociedade, objetivando uma atuação transformadora voltada para os interesses e necessidades da maioria da população e implementadora de desenvolvimento regional e de políticas públicas, tendo o comprometimento efetivo com a solução dos problemas e desafios de seu contexto econômico e social (FARIA, 2001).

Este estudo, partindo deste pressuposto, está pautado em uma reflexão sobre Extensão Universitária, a partir de suas políticas, ações e diretrizes, considerando a sua importância tanto para as Instituições de Ensino Superior (IES) quanto para a Sociedade. Para tal reflexão, foi escolhido o projeto de Extensão Teste da Orelhinha em Irati e Região (TOIR), por se tratar de uma proposta de prestação de serviços aos neonatos e lactentes nascidos em nove municípios pertencentes à $4^{\mathrm{a}}$ Regional de Saúde do Estado do Paraná, tendo como objetivo principal a identificação precoce da perda auditiva.

A relevância de uma pesquisa sobre essa temática está em verificar se os conhecimentos produzidos nas IES, por meio do ensino, da pesquisa e da extensão, potencializam benefícios na região em que são desenvolvidos, propondo uma discussão sobre as relações que a Universidade mantém com a Sociedade, bem como da dinâmica das políticas públicas, ações e diretrizes institucionais de Extensão Universitária.

Os objetivos deste estudo foram a identificação dos benefícios do referido projeto de Extensão às comunidades atendidas, pautado em uma reflexão sobre a relação da Universidade com a Sociedade, e a verificação da percepção da comunidade, dos professores, dos acadêmicos e dos gestores municipais de saúde com relação ao conhecimento que possuem sobre Extensão Universitária e o Projeto TOIR.

\section{METODOLOGIA}

Os dados relativos ao Projeto TOIR compreenderam o período entre os anos de 2007 e 2013. Foi desenvolvido um estudo de caso com abordagem qualitativa, onde o objeto, neste caso o Projeto TOIR, 
é uma unidade analisada em profundidade, visando o exame detalhado e intensivo de um ambiente, de uma amostra populacional ou de uma situação em particular, a partir da perspectiva das pessoas nele envolvidas, considerando todos os pontos de vista relevantes (GODOY, 1995).

Para o levantamento da percepção da comunidade, dos professores, dos acadêmicos e dos gestores municipais de saúde foram utilizados questionários semiestruturados, diferenciados de acordo com a linguagem utilizada e temáticas abordadas, considerando as características de cada população investigada e a relação com o projeto. A coleta dos dados foi realizada nas dependências da Clínica Escola de Fonoaudiologia (CEFONO), da Universidade Estadual do Centro Oeste (UNICENTRO), no município de Irati (PR) e nas Secretarias Municipais de Saúde, mediante autorização no Termo de Consentimento Livre e Esclarecido, aprovado pelo Comitê de Ética e Pesquisa da Universidade do Vale do Itajaí, sob o parecer de $n^{\circ} 423.438$.

Participaram da amostra a comunidade e gestores municipais de saúde de três municípios. 0 critério de escolha, seguindo a orientação de Rodrigues (2012), foi a relação percentual acima de $90 \%$, entre bebês triados e bebês nascidos vivos, de cada município no ano de 2011. 0 município de Fernandes Pinheiro apresentou 95,5\% de adesão ao projeto, Rio Azul apresentou 94,7\% e Irati, 91,3\%, sendo as cidades escolhidas para a pesquisa por apresentarem os maiores índices de participação no projeto TOIR. Os respondentes da comunidade foram a mãe ou o pai dos bebês atendidos pelo Projeto.

Os acadêmicos do $4^{\circ}$ ano do curso de Fonoaudiologia da UNICENTRO responderam a entrevista em função do projeto TOIR ser desenvolvido na disciplina obrigatória de Estágio Supervisionado em Audiologia II. Os docentes entrevistados foram aqueles que supervisionavam os alunos do projeto e ex-professores que já não faziam parte do corpo docente da UNICENTRO, mas que haviam atuado como supervisores no estágio.

Um total de 61 questionários foram respondidos, sendo que 30 foram aplicados à comunidade residente nos três municípios citados, 24 aos acadêmicos do $4^{\circ}$ ano do curso de Fonoaudiologia no ano de 2013, quatro aos docentes e três aos Secretários de Saúde. A aplicação do questionário ocorreu de três formas. Os acadêmicos receberam o questionário impresso, com as recomendações e instruções por escrito. Os docentes receberam por correio eletrônico, incluindo as recomendações e instruções. A comunidade atendida no Projeto e os gestores municipais de saúde responderam o questionário verbalmente, aplicado em forma de entrevista.

Os questionários contemplaram variáveis estruturadas e semiestruturadas. Para facilitar a compreensão das informações e a discussão dos resultados, foi utilizada a técnica do agrupamento dos três protocolos aplicados para, então, serem selecionadas 12 categorias de avaliação ${ }^{5}$, considerando a proximidade das perguntas, organizadas para facilitar a análise e discussão dos resultados com base na Política Nacional de Extensão Universitária (2012) e no Regulamento de Extensão da UNICENTRO (2012).

51. Perfil do entrevistado; 2. Conhecimento sobre Extensão Universitária; 3. Propostas extensionistas da UNICENTRO; 4. Acesso à participação e informação; 5 . Conhecimento sobre o projeto TOIR; 6. Vínculo com o projeto TOIR; 7. Conhecimento sobre a CEFONO / UNICENTRO; 8. Dificuldades na execução do projeto TOIR; 9. Apoio dos Municípios na execução do projeto TOIR; 10. Melhoria da saúde e da qualidade de vida da população da região; 11. Impacto e transformação social; 12. Elaboração de política pública. 
Algumas variáveis quantitativas discretas, por serem de natureza numérica, foram mensuradas conforme a escala tipo Likert de quatro pontos (nunca, às vezes, frequentemente e sempre, com valores de 1, 2, 3 e 4, respectivamente), por considerar que professores, acadêmicos e gestores teriam certo conhecimento dos itens do questionário. Para as categorias de avaliação das questões semiestruturadas do protocolo de entrevista, utilizou-se a técnica da análise de conteúdo da percepção dos participantes, buscando verificar as diferenças e as semelhanças entre suas respostas e os preceitos estabelecidos pela Política Nacional de Extensão Universitária e pelo Regulamento de Extensão da UNICENTRO, em relação à concepção, princípios, diretrizes e objetivos da Extensão Universitária.

A estatística descritiva foi usada tendo em vista que a amostra é reduzida (31 questionários), dividida em 4 grupos heterogêneos (30 da comunidade, 24 acadêmicos, 4 docentes e 3 gestores, com dois tipos distintos de questionários), o que não possibilitou a realização da estatística paramétrica, que concentra a maior parte das análises/testes estatísticos. Além disso, utilizou-se de uma escala não adequada ao uso de análises paramétricas. Toda a análise descritiva foi realizada por meio do programa Excel.

\section{RESULTADOS}

A amostra total foi composta de 61 participantes, sendo 57 do sexo feminino e quatro do sexo masculino. Dos 30 participantes dos três municípios pertencentes à comunidade atendida no projeto, foram entrevistados 28 mães e dois pais, sendo o maior número $(n=11)$ da cidade de Rio Azul. A maioria dos bebês nasceu na Santa Casa de Irati (53,33\%; $n=16)$. Isso se deve ao fato do município de Irati ser sede do Consórcio Intermunicipal de Saúde e possuir um hospital com melhores condições de pessoal e equipamentos, além da Unidade de Terapia Intensiva (UTI) neonatal, em relação aos demais hospitais da região.

Professores, acadêmicos e gestores de saúde resultaram em $50,82 \%(n=31)$ dos respondentes. A percepção de professores, acadêmicos e gestores de saúde, quanto ao entendimento sobre Extensão Universitária, revelou conhecimento satisfatório sobre a indissociabilidade entre ensino, pesquisa e extensão conforme relato apresentado abaixo:

São atividades ou projetos que relacionam os conteúdos / programas aprendidos na Universidade com a comunidade. Envolve, atende e orienta a população, os professores e os alunos" (Docente A). "São projetos que realizam ações que irão beneficiar a comunidade, além de promover discussões teóricas coordenadas por professores supervisores" (Acadêmico E). "Um bem que os alunos proporcionam para uma determinada localidade em específica área de conhecimento (Gestor C).

As respostas semelhantes dos entrevistados foram sintetizadas e agrupadas em categorias para facilitar a compreensão do conteúdo a partir de uma breve análise descritiva, conforme Tabela 1. 
Tabela 1 - Respostas dos docentes, acadêmicos e gestores referentes à concepção sobre Extensão Universitária $(n=31)$

Pergunta: $\mathbf{O}$ que você entende por Extensão Universitária?

1

2

Curso de capacitação universitária ou progressão que aprimoram o conhecimento.

Modalidade acadêmica onde aluno e professor pesquisam e desenvolvem atividades relacionadas a uma área específica.

Projetos vinculados ao ensino e à pesquisa que aproximam a Universidade da comunidade, por meio do acesso à prestação de serviços de qualidade e sem custos, trazendo conhecimentos ao aluno.

Atividades extracurriculares que complementam as da graduação.

União da teoria com a prática.

Atividades realizadas fora da Universidade.

Tripé: ensino, pesquisa e extensão. n $\%$

2

6,45

Fonte: Autora.

Aos gestores de saúde foi questionado se conheciam as propostas extensionistas desenvolvidas na UNICENTRO, Campus de Irati. Os três entrevistados responderam de forma afirmativa, elencando o Teste da Orelhinha, além de outros acompanhamentos que a Fonoaudiologia realiza na Clínica Escola.

$\mathrm{Na}$ Tabela 2, com relação ao conhecimento das características das propostas extensionistas, é possível verificar que a análise da escala Likert revelou médias abaixo de 3,50, tendo sido apresentadas as respostas "às vezes" e "frequentemente" para os itens 3 a 8. A articulação dos saberes nas diferentes áreas do conhecimento, o impacto e a transformação social da comunidade, a superação de desigualdades, bem como a contribuição para a elaboração de políticas públicas a partir de uma interação dialógica entre a universidade e os setores sociais não são efetivamente incorporados nas propostas extensionistas.

Para os itens 1 e 2, as médias foram superiores, indicando que os entrevistados "frequentemente" ou "sempre" têm percebido estas características das diretrizes nas ações que conhecem. 0 primeiro item, específico para o projeto de extensão Teste da Orelhinha, apresentou respostas favoráveis à relação dos três níveis - ensino, pesquisa e extensão. A contribuição para a formação acadêmica foi percebida como sendo relevante, apresentando a maior média na análise das respostas $(3,94)$.

De acordo com a proximidade dos valores de desvio padrão, foi possível considerar que o nível de coesão é relativamente próximo entre os respondentes em cada uma das questões. 
Tabela 2 - Respostas dos docentes, acadêmicos e gestores referentes ao conhecimento das características das propostas extensionistas da UNICENTRO $(n=31)$

Pergunta: Quanto às propostas extensionistas da UNICENTRO, Campus de Irati, você percebe que:

$1 \quad$ O TOIR se relaciona com o ensino, a pesquisa e a extensão?

n Média $\begin{array}{r}\text { Desvio } \\ \text { padrão }\end{array}$

2 Contribuem para a formação acadêmica dos estudantes?

31

3,94

0,25

3
Propiciam a articulação de saberes entre diferentes áreas do conhecimento?

31

3,29

0,69

4

Proporcionam impacto e transformação social da comunidade?

Contribuem para a elaboração de políticas públicas comprometi6 das com o desenvolvimento solidário, democrático e sustentável? versidade e outros setores da Sociedade?

Fonte: Autora.

A Tabela 3 mostra as respostas de docentes e acadêmicos com relação à coordenação/participação em atividades extensionistas desenvolvidas no Campus. Conforme análise da escala Likert, a média dos respondentes está localizada, segundo a escala intervalar utilizada, entre as respostas "nunca" e "às vezes" para os itens 3 e 4. A participação dos acadêmicos bem como a coordenação dos docentes é menor para as modalidades de curso e eventos de extensão. As respostas "às vezes" e "frequentemente" foram mais selecionadas para participação ou coordenação de ações extensionistas que contemplam intervenções na comunidade. 
Tabela 3 - Respostas de professores e acadêmicos com relação à participação em atividades extensionistas no Campus de Irati $(n=28)$

\begin{tabular}{|c|c|c|c|c|}
\hline 1 & $\begin{array}{c}\text { Recebe(eu) informações sobre a extensão } \\
\text { quando ingressou na Universidade? }\end{array}$ & 28 & 2,07 & 0,86 \\
\hline 2 & Participa(ou) ou coordena(ou) ações extensionistas? & 27 & 2,85 & 1,23 \\
\hline 3 & Participa(ou) ou coordena(ou) cursos de extensão? & 28 & 1,93 & 1,12 \\
\hline 4 & Participa(ou) ou coordena(ou) eventos de extensão? & 27 & 1,67 & 0,96 \\
\hline 5 & $\begin{array}{c}\text { Participa(ou) ou coordena(ou) alguma atividade de prestação de } \\
\text { serviço extensionista? }\end{array}$ & 28 & 2,50 & 1,20 \\
\hline
\end{tabular}

Fonte: Autora.

Dos 24 acadêmicos e quatro professores entrevistados, apenas dois responderam que "sempre" receberam informações sobre extensão, seja por parte do seu Departamento ou por meio da Comunicação Social interna à comunidade acadêmica

A comunidade e os gestores municipais de saúde foram indagados sobre a percepção que possuem em relação à proposta extensionista Teste da Orelhinha em Irati e Região. Dos 33 entrevistados, sendo 30 da comunidade e três gestores, todos já ouviram falar e $30,30 \%(n=10)$ soube do Teste da Orelhinha no acompanhamento do pré-natal. Outros sete participantes conheceram o teste por intermédio de familiares ou outras pessoas. A comunidade demonstrou conhecimento sobre o objetivo do Teste da Orelhinha, sendo que $70 \%$ dos respondentes respondeu que a finalidade do TOIR é verificar se o bebê escuta bem.

“Ouvi por outras mães” (Mãe A). “[...] na maternidade” (Mãe C). “[...] pela minha tia quando teve bebê” (Mãe N). "Secretaria Municipal de Saúde” (Gestor A). “[...] Hospital” (Gestor B). "O Teste serve para verificar a audição do bebe" (Mãe G). "Serve para detectar problemas na audição. Tudo o que é detectado no início é mais fácil de diagnosticar” (Mãe L).

Ao questionar aos professores, acadêmicos e gestores se os objetivos propostos pelo TOIR estão sendo atingidos com êxito, a maioria dos acadêmicos ( $n=23 ; 95,83 \%)$ respondeu que "frequentemente" e "sempre". A resposta dos docentes foi mais crítica, pois dois deles (50\%) consideram que "às vezes" os objetivos são alcançados, tendo muitas variáveis a serem analisadas neste quesito. Um dos gestores de saúde não conhece os objetivos do projeto TOIR e um afirmou que o Teste da Orelhinha "sempre" atinge os objetivos propostos.

Uma porcentagem de $60 \%(n=18)$ dos entrevistados da comunidade não respondeu ao questionamento sobre a contribuição do Projeto TOIR para a elaboração e efetivação de políticas públicas. Contudo, $23,33 \%(n=7)$ relatou que o Teste da Orelhinha pode melhorar a qualidade de vida das crianças e das famílias, visto que, promove a detecção precoce da perda auditiva, assim como pode ser um instrumento de superação de desigualdades e da exclusão. Entretanto, 80,64\% dos docentes, 
acadêmicos e gestores $(n=31)$ acreditam que "frequentemente" e "sempre", o projeto contribui para o desenvolvimento de políticas públicas voltadas para a área da saúde, apesar de $77,41 \%$; $(n=31)$ não soube explicar de que forma esse processo acontece.

Os professores e acadêmicos participantes da pesquisa acreditam, em sua maioria (78,57\%; n= 22), que "às vezes" e "frequentemente" há o envolvimento e apoio dos gestores municipais no projeto TOIR. Anualmente, a coordenação do projeto realiza reuniões com os gestores e técnicos para reforçar a importância da parceria entre secretarias municipais e universidade, a fim de intensificar a disseminação do Teste da Orelhinha à comunidade. A percepção de professores, acadêmicos e gestores de saúde ( $n=21 ; 67,74 \%$ ) é de que o projeto TOIR é "sempre" um instrumento de mudança em busca da melhoria da qualidade de vida e da superação de desigualdades e da exclusão das crianças, por meio da detecção precoce da perda auditiva, gerando impacto social.

Os profissionais que coordenam o projeto TOIR não têm acesso aos resultados dos encaminhamentos realizados para avaliação otorrinolaringológica, pois não existe um retorno satisfatório por parte de outros segmentos da área da saúde quanto aos procedimentos realizados, aos encaminhamentos e resultados, impedindo uma maior efetividade no desenvolvimento do programa. Uma porcentagem de $35,48 \%$ ( $n=11$ ) dos entrevistados não souberam responder de que forma o projeto pode proporcionar uma transformação e impacto social.

\section{DISCUSSÃO}

A análise trazida pela Tabela 1 e a partir dos relatos dos respondentes caracteriza o projeto de extensão Teste da Orelhinha como uma ação que reafirma a Extensão Universitária como um mecanismo por meio do qual se estabelece a inter-relação da Universidade com a Sociedade, com vistas a uma atuação transformadora, voltada para os interesses e necessidades da maioria da população e propiciadora do desenvolvimento social e regional, assim como para o aprimoramento das políticas públicas (FORPROEX, 2012). Nesse sentido, é importante reiterar que a Extensão Universitária almeja produzir benefícios não apenas para a sociedade e a Universidade, mas também promover uma mudança de percepção entre os discentes, apresentando diferentes realidades e a vivência de experiências humanísticas fundamentais para um profissional que auxilia no desenvolvimento das pessoas e das comunidades que pertencem.

Valentim e outros autores (2003) afirmam que ao procurar colocar o conhecimento produzido a serviço da clientela, contribuindo para que ela tome consciência da totalidade da realidade e do processo histórico em que está inserida, projetos de extensão à comunidade buscam intermediar a apropriação do conhecimento por parte dos docentes e discentes e dinamizar os processos de difusão capazes de proporcionar o desenvolvimento de suas propostas pedagógicas. Porém, ainda foram evidentes algumas limitações em relação a execução do projeto deste estudo, começando pelos acadêmicos, os quais, mesmo fazendo parte de um projeto extensionista, ainda demonstram um conhecimento superficial sobre 0 conceito de Extensão Universitária, conforme resultados apresentados na Tabela 2. 
O curso de Fonoaudiologia da UNICENTRO é o único em Instituição Pública de Ensino Superior no Paraná. Tal caráter público tem implicações importantes para o perfil da profissão de fonoaudiólogo e dentre as competências necessárias, está aquela destinada à atuação nos serviços de saúde. Nesse processo de formação, a participação em programas de extensão universitária amplia as possibilidades de aprendizado por permitir a vivência entre os ambientes acadêmico e comunitário. Desse modo, o curso tende a se comprometer com as atividades voltadas, essencialmente para a saúde pública por meio de ações extensionistas, conforme resultados apresentados na Tabela 3.

De acordo com Moura e outros autores (2001), a extensão universitária visa a levar, por um lado, às comunidades carentes, o desenvolvimento e a aplicação de pesquisas e ensinos realizados em seus departamentos acadêmicos, buscando modificar realidades e melhorar a qualidade de vida das populações assistidas. Por outro lado, abre a convivência e a interação com as comunidades e, no convívio, novos conhecimentos são descobertos e situações diferentes daquelas vivenciadas nas clínicas intramuros acontecem; constrói-se, dessa forma, uma pluralidade que flexibiliza a elaboração de projetos de pesquisas e fortalece o ensino que ali se constituem.

$\mathrm{Na}$ análise sobre as percepções dos respondentes em relação aos benefícios promovidos pelo Teste da Orelhinha, constatou-se que a detecção precoce da perda auditiva antes de a criança completar três meses de idade é o principal benefício do teste, além da informação e conscientização das famílias quanto à importância de avaliar a audição de seus filhos. 0 diagnóstico da perda auditiva e a intervenção precoce no primeiro ano de vida possibilitam a estimulação no período ideal ao neonato. Isso devido à maturação do Sistema Nervoso Central, que segundo a teoria moderna da neuroplasticidade, passa por um período ideal, no qual os sinais auditivos são otimamente recebidos e utilizados para atividades pré-linguísticas importantes (SOUZA, 2015).

Segundo a Organização Mundial de Saúde (WHO, 2010), a perda auditiva em crianças em todo o mundo constitui um obstáculo particularmente relevante para um satisfatório desenvolvimento e educação, incluindo a aquisição da linguagem. De acordo com estudos conduzidos em diferentes países, de 0,5 a cada 5 de 1000 neonatos e crianças apresentam uma perda auditiva congênita ou perda auditiva sensorioneural na primeira infância ou uma surdez de grau severo a profundo. Ressalta ainda que a efetividade de muitos programas de triagem e acompanhamento de neonatos e crianças não está vinculada à tecnologia e sim à infraestrutura e capacidade dos recursos humanos em prover o serviço de intervenção e suporte para as famílias, como é o caso do Projeto deste estudo, cuja reflexão da percepção dos entrevistados destacou que o Teste da Orelhinha pode ser um instrumento de mudança em busca da melhoria da qualidade de vida das crianças e das famílias da região e da superação de desigualdades e exclusão.

Esse processo ocorre, de acordo com os relatos, por meio da detecção precoce da perda auditiva, do acompanhamento da saúde do bebê, da informação aos sujeitos, da obrigatoriedade da Lei (BRASIL, 2010), do atendimento igualitário, pelo conhecimento das consequências e implicações da perda auditiva na vida daqueles que a possuem, bem como a pais e gestores de saúde e, pela maior infiltração do projeto na sociedade, proporcionando a informação sobre a importância da audição para o desenvolvimento global das crianças. 
Françozo e outros autores (2010) ressaltam que o acompanhamento sistemático às famílias dos lactentes que não passam na primeira avaliação e a conscientização destas sobre a detecção precoce da perda auditiva e suas consequências podem contribuir para o aumento da taxa de adesão na segunda etapa da triagem. Os depoimentos deste estudo revelam que a prestação de serviço gratuito para a comunidade de Irati e região é a contribuição do Teste da Orelhinha para a elaboração e efetivação de políticas públicas. Este projeto faz parte de tantos outros presentes em 24 dos 27 estados brasileiros, sendo que o Brasil tem um dos maiores e mais antigos programas de TAN da América Latina, segundo Cavalcanti e Guerra (2012).

A preocupação em conscientizar a população para os problemas de audição na infância e a necessidade de detecção e intervenção precoce é bem mais anterior, com a criação do Grupo de Apoio a Triagem Auditiva Neonatal Universal (GATANU, 2013), em 1988, primeiro órgão destinado a TAN. A partir de então, em 2004, o Ministério da Saúde criou a Política Nacional de Atenção à Saúde Auditiva com ações de baixa, média e a alta complexidade, além de terapia fonoaudiológica e a Lei Federal no 12.303 de 2/08/2010 tornou obrigatória a realização gratuita do exame denominado Emissões Otoacústicas Evocadas, em todos os hospitais e maternidades, nas crianças nascidas em suas dependências (BRASIL, 2010).

Em 2012, foram publicadas as “Diretrizes de Atenção da Triagem Auditiva Neonatal” (BRASIL, 2012), com objetivo de oferecer orientações às equipes multiprofissionais para o cuidado da saúde auditiva na infância, em especial à TAN, nos diferentes pontos de atenção da rede.

Em estudo pioneiro no que diz respeito ao olhar sobre a rede de Atenção à Saúde Auditiva Neonatal no Município do Rio de Janeiro, Lima e outros autores (2015) verificaram que as três unidades materno-infantis escolhidas para o estudo realizavam triagem auditiva neonatal, porém não conseguem atender toda a demanda, inferindo que a situação da TAN no município precisa de ajustes para melhor contribuir com a saúde pública. No período do estudo, $80,2 \%$ dos nascidos vivos nas maternidades municipais do Rio de Janeiro não realizaram triagem auditiva neonatal, revelando um percentual baixo de realização da TAN, aquém do indicado pelo Comitê Multiprofissional em Saúde Auditiva (LEWIS et al., 2007). Este estudo mostra uma realidade bastante diferente comparativamente ao serviço prestado pelo Projeto TOIR, o qual por meio de uma ação extensionista desempenha um papel importantíssimo na detecção da perda auditiva em nascidos vivos de nove municípios pertencentes à $4^{\text {a }}$ Regional de Saúde do Estado do Paraná.

Nos anos de 2007 e 2008, em média, $60 \%$ dos nascidos vivos na região foram triados no Projeto TOIR. Em 2009 e 2010 essa média ficou em torno de 70\% e em 2011 foram atingidos, em média, $83,5 \%$, estando ainda abaixo do esperado para obter um indicador de qualidade do programa, mesmo tendo apresentado um aumento de $11,5 \%$ na adesão das famílias à triagem auditiva com relação aos quatro anos anteriores (MAGNI et al., 2013). Apesar de não se caracterizar como programa de triagem auditiva neonatal universal, apresentou um índice de encaminhamentos para diagnóstico de apenas $3,6 \%$, o que pode ser considerado como um indicador de qualidade para o programa, conforme indicação do Comitê Multiprofissional em Saúde Auditiva (LEWIS et al., 2007).

As políticas públicas direcionadas às Instituições de Ensino Superior são, geralmente, específicas às questões do ensino ou da pesquisa. Os gestores ainda possuem pouca compreensão sobre o potencial 
de contribuição que as ações extensionistas têm quanto à possibilidade de desenvolvimento de políticas públicas direcionadas à educação, saúde, trabalho, emprego e renda, havendo a necessidade de um estreitamento dos laços e ampliação da divulgação das ações extensionistas em prol da Sociedade.

Os docentes, entretanto, são mais críticos em responder que “às vezes” os objetivos são alcançados, já que existem muitas variáveis a serem analisadas neste quesito, como, por exemplo, associações entre os indicadores socioeconômicos e as condições de saúde da população, bem como o grau de escolaridade da família (GRIZ et al., 2015), já que a educação formal é um fator importante para ser levado em conta na atuação com a população, quanto às práticas de promoção, proteção e recuperação da saúde, incluindo a saúde auditiva, pois determina uma melhor condição de vida, um melhor acesso a condições favoráveis de saúde e melhores perspectivas futuras para a saúde da mãe e de seu filho.

0 reflexo disso fica evidente na falta de informação sobre saúde auditiva infantil na formação dos profissionais de enfermagem e a separação entre o ensino e prática, o que favorece o desconhecimento dos indicadores de risco para perda auditiva (IRPA) e, consequentemente, a não valorização da triagem auditiva às famílias. No estudo de Barbosa e outros autores (2013), foi observado que mais de $60 \%(64,6 \%)$ da equipe de enfermagem de um hospital universitário conhecia algum IRPA, porém após ações educativas com base nas informações coletadas em um questionário, com base nos preceitos da metodologia de Paulo Freire, o conhecimento foi ampliado, sendo citado por $98,8 \%$ dos participantes.

Nesse sentido, é necessário e urgente reconhecer as atividades de extensão como elos entre as atividades desenvolvidas nas universidades e a sociedade em geral, pois mais do que atender às demandas sociais e a estas prestar contas, as atividades de extensão são também produtoras de conhecimentos, norteando ações de educação continuada entre profissionais de saúde, visando à intervenção, iniciada o mais cedo possível, minimizando, assim, as consequências da perda auditiva na vida do indivíduo, de seus familiares e do Estado.

Faz-se necessário ampliar e aperfeiçoar os serviços de Triagem Auditiva Neonatal (TAN) para abranger toda demanda regional e atingir, conforme preconizado pelo Joint Committee on Infant Hearing $(\mathrm{JClH}, 2007)$, um percentual superior a 95\% do total de nascidos vivos que devem ser submetidos à triagem até o primeiro mês de vida e intervenção fonoaudiológica o mais rapidamente possível após o diagnóstico e, preferencialmente, no máximo até o sexto mês de vida (LEWIS, 1987; YOSHINAGA-ITANO, 2003).

Cabe ressaltar que a intervenção realizada no âmbito de um projeto de Extensão Universitária não tem o intuito de substituir as funções do Estado, mas por meio das ações produzir conhecimento científico, cultural, filosófico e político de forma interdisciplinar, tornando estes conhecimentos acessíveis às comunidades e promovendo a interação transformadora entre Universidade e sociedade.

\section{CONCLUSÃO}

O estudo averiguou que o principal benefício do projeto TOIR é a identificação da perda auditiva antes de a criança completar três meses de idade além de estabelecer uma relação entre a Universidade e outros 
setores da Sociedade, com vistas a uma atuação transformadora, voltada para os interesses e necessidades da maioria da população e implementadora de desenvolvimento regional e de políticas públicas.

Os resultados obtidos poderão nortear a equipe de profissionais para as devidas ações de intervenção, na tentativa de ampliar a informação e conscientização das famílias quanto à importância de avaliar a audição de seus filhos e, principalmente, de ampliar o acesso àqueles que tiverem sido diagnosticados como portadores de uma deficiência auditiva em algum grau, para que possam ser encaminhados a uma intervenção reabilitativa e educacional apropriada e acompanhados, juntamente com suas famílias, por um programa assistencial. Estas ações e estratégias deverão visar um maior envolvimento das Secretarias Municipais de Saúde, na elaboração e implementação de políticas públicas voltadas para a área da saúde, com o intuito de melhorar a execução do projeto e a qualidade de vida da comunidade beneficiada.

A Extensão Universitária é imprescindível à democratização do conhecimento, visto que, produz benefícios não apenas para a sociedade e a Universidade, mas também promove uma mudança de percepção entre os discentes, apresentando diferentes realidades e a vivência de experiências humanísticas fundamentais para um profissional que auxilia no desenvolvimento das pessoas e das comunidades que pertencem.

\section{REFERÊNCIAS}

BARBOSA, Camila Padilha et al. Newborn and infant hearing health education for nursing professionals. Braz J Otorhinolaryngol., v. 79, n. 2, p. 226-232, 2013.

BRASIL. MINISTÉRIO DA SAÚDE n0 12.303/2010. Dispõe sobre a obrigatoriedade de realização do exame denominado Emissões Otoacústicas Evocadas. Diário Oficial da União. Brasília, 03 ago 2010.

CAVALCANTI HG, Guerra RO. The role of maternal socioeconomic factors in the commitment to universal newborn hearing screening in the northeastern region of Brazil. Int J Pediatr Otorhinolaryngol., v. 76, p. 1661-1667, 2012.

FARIA, Dóris Santos de. Construção conceitual da extensão universitária na América Latina. Brasília: UNB, 2001.

FÓRUM DE PRÓ-REITORES DE EXTENSÃO das universidades públicas brasileiras e secretaria de educação superior do ministério de educação. Plano Nacional de Extensão Universitária. Edição Atualizada 2012.

FRANÇOZO, Maria de Fátima de Campos et al. Adesão a um Programa de Triagem Auditiva Neonatal. Saúde Soc., São Paulo, v. 19, n. 4, p. 910-918, 2010. 
GODOY, Arilda Schmidt. Pesquisa Qualitativa tipos fundamentais. Revista de Administração de Empresas, São Paulo, v. 35, n. 3, p. 20-29, 1995.

GRIZ, Silvana Maria Sobral et al. Análise dos aspectos socioeconômicos e demográficos de famílias atendidas em um Programa de Triagem Auditiva Neonatal ao longo de três anos. Rev. CEFAC, v. 17 (Supl1), p. 88-95, 2015.

GRIZ, S. M. S., BARBOSA, C. P.; SILVA, A. R. A.; RIBEIRO, M. A.; MENEZES, D. C. Aspectos demográficos e socioeconômicos de mães atendidas em um programa de triagem auditiva neonatal. Rev. Soc. Bras. Fonoaudiol., v. 15, n. 2, p. 179-183, 2010.

Grupo de Apoio a Triagem Auditiva Neonatal Universal - GATANU, out. 2013.

JCIH - Joint Committee on Infant Hearing. Year 2007. Position Statement: principles and guidelines for early hearing detection and intervention programs. Pediatrics, v. 120, n. 4, p. 898-921, 2007.

LEWIS, D. R. Audição: um procedimento de avaliação para crianças de 6 meses a 2 anos de idade. ‘987. Dissertação (Mestrado) - Pontifícia Universidade Católica de São Paulo, 1987.

LEWIS, D. R.; MARONE, S. A. M.; MENDES, B. C. A.; CRUZ, O. L. M.; NÓBREGA, M. COMUSA-Comitê de Programa de Saúde Auditiva em duas maternidades públicas. Rev Soc Bras Fonoaudiol., v. 12, n. 2, p. 99-105, 2007.

LIMA, Priscila Tavares et al. A triagem auditiva neonatal na Rede Municipal do Rio de Janeiro, Brasil, Ciência Saúde Coletiva, v. 20, n. 1, 2015.

MAGNI, C; DE CONTO, J.; LUDERS, D.; AMARAL, M. I.R. Teste da orelhinha em Irati e região: perfil dos bebês triados no teste da orelhinha nos anos de 2011 e 2012. Seminário Paranaense de Fonoaudiologia, 4, Irati, PR, 2013.

MOURA, L. F. A. D.; LIRA, D. M. M. P.; MOURA, M. S., BARROS, S. S. L. V.; LOPES T. S. P.; LEOPOLDINO, V. D. et al. Apresentação do Programa Preventivo para Gestantes e Bebês. J Bras de Odontopediatr Odontol Bebê, v. 4, n. 17, p. 10-14, 2001.

RODRIGUES, Daniela Tôtolo. Teste da Orelhinha: Perfil dos bebês triados em um programa do Centro Sul do Paraná. Monografia, Universidade Estadual do Centro-Oeste, UNICENTRO, Irati, 2012.

SOUZA, Gabriele Libano de. Características Audiológicas relacionadas ao baixo peso, prematuridade, anóxia/hipóxia e infecções congênitas ao nascimento: da triagem auditiva 
neonatal ao diagnóstico. 2015. Tese (Doutorado)

- Universidade Estadual de Campinas, UNICAMP,

Campinas, SP, 2015.

UNICENTRO - Universidade Estadual do CentroOeste. Resolução Conjunta n0 007-CEPE-CAD/

UNICENTRO: Aprova o Regulamento das Atividades

Extensionistas da UNICENTRO, Guarapuava, 2012.

VALENTIM, Marta Ligia Pomim et al. As

Articulações da pesquisa com o ensino e a extensão nos cursos de Biblioteconomia e Ciência da Informação no Mercosul. V. 15, n. 2, p. 105-117, 2003.

YOSHINAGA-ITANO, C. From Screening to Early identification and Intervention: Discovering predictors to successful outcomes for children with significant hearing loss. J. Deaf Stud Deaf Educ., v. 8, n. 11, p. 1-30, 2003.

WHO. World Health Organization. Newborn and infant hearing screening. Geneva, 2010.

1 Mestre em Gestão de Políticas Públicas pela Universidade do Vale do Itajaí - SC. E-mail: karla_nadal@hotmail.com

2 Doutor em História; Professor da Universidade do Vale do Itajaí - SC. E-mail: paulo_rmo@hotmail.com

3 Professora do Departamento de Fonoaudiologia e do Programa de Pós-Graduação em Desenvolvimento Comunitário da Universidade Estadual do Centro-Oeste. E-mail: crismagni@unicentro.br

4 Professor do Departamento de Ciências Contábeis e do Programa de Pós-Graduação em Administração da Universidade Estadual do Centro-Oeste. E-mail: marcosrobertokuhl@yahoo.com.br

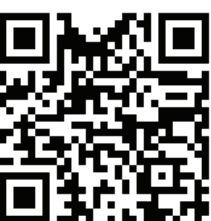

A autenticidade desse artigo pode ser conferida no site https://periodicos. set.edu.br

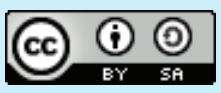

Este artigo é licenciado na modalidade acesso abertosob a Atribuição-Compartilhalgual CC BY-SA

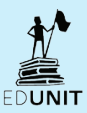


